\title{
Liquid bridge length scale based nondimensional groups for mapping transitions between regimes in capillary break-up experiments
}

\author{
Citation for published version (APA): \\ Verbeke, K., Formenti, S., Briatico-Vangosa, F., Mitrias, C., Reddy, N. K., Anderson, P. D., \& Clasen, C. (2020). \\ Liquid bridge length scale based nondimensional groups for mapping transitions between regimes in capillary \\ break-up experiments. Physical Review Fluids, 5(5), [051901]. https://doi.org/10.1103/PhysRevFluids.5.051901
}

DOI:

10.1103/PhysRevFluids.5.051901

Document status and date:

Published: 01/05/2020

Document Version:

Publisher's PDF, also known as Version of Record (includes final page, issue and volume numbers)

\section{Please check the document version of this publication:}

- A submitted manuscript is the version of the article upon submission and before peer-review. There can be important differences between the submitted version and the official published version of record. People interested in the research are advised to contact the author for the final version of the publication, or visit the DOI to the publisher's website.

- The final author version and the galley proof are versions of the publication after peer review.

- The final published version features the final layout of the paper including the volume, issue and page numbers.

Link to publication

\footnotetext{
General rights

- You may freely distribute the URL identifying the publication in the public portal. follow below link for the End User Agreement:

www.tue.nl/taverne

\section{Take down policy}

If you believe that this document breaches copyright please contact us at:

openaccess@tue.nl

providing details and we will investigate your claim.
}

Copyright and moral rights for the publications made accessible in the public portal are retained by the authors and/or other copyright owners and it is a condition of accessing publications that users recognise and abide by the legal requirements associated with these rights.

- Users may download and print one copy of any publication from the public portal for the purpose of private study or research.

- You may not further distribute the material or use it for any profit-making activity or commercial gain

If the publication is distributed under the terms of Article $25 \mathrm{fa}$ of the Dutch Copyright Act, indicated by the "Taverne" license above, please 


\title{
Liquid bridge length scale based nondimensional groups for mapping transitions between regimes in capillary break-up experiments
}

\author{
Karel Verbeke, ${ }^{1, *}$ Susanna Formenti, ${ }^{1,2,}{ }^{*}$ Francesco Briatico Vangosa $\odot,{ }^{2}$ Christos Mitrias, ${ }^{3}$ \\ Naveen Krishna Reddy, ${ }^{4,5}$ Patrick D. Anderson ${ }^{0},{ }^{3}$ and Christian Clasen ${ }^{1}{ }^{1}$ \\ ${ }^{1}$ Department of Chemical Engineering, KU Leuven, Celestijnenlaan 200f, 3001 Leuven, Belgium \\ ${ }^{2}$ Department of Chemistry, Materials and Chemical Engineering "Giulio Natta", Politecnico di Milano, \\ Piazza L. da Vinci 32, 20133 Milano, Italy \\ ${ }^{3}$ Department of Mechanical Engineering, Eindhoven University of Technology, P.O. Box 513, \\ 5600 MB Eindhoven, The Netherlands \\ ${ }^{4}$ Hasselt University, Martelarenlaan 42, 3500 Hasselt, Belgium \\ ${ }^{5}$ IMO-IMOMEC, Wetenschapspark 1, 3590 Diepenbeek, Belgium
}

(Received 15 August 2019; accepted 1 April 2020; published 1 May 2020)

\begin{abstract}
Criteria to identify transitions between dynamic self-similar linear thinning regimes of liquid bridges are of utmost importance in order to accurately interpret results in capillary break-up rheometry. Currently available criteria encompass many experimental difficulties or rely on numerical approaches. Here, we introduce a different set of nondimensional groups, $\mathrm{Oh}_{\mathrm{L}}=\eta_{\text {in }} / \sqrt{\gamma \rho L}$ and $a=R / L$, based on the experimentally relevant axial length scale of a liquid bridge $L$, for viscous-dominated fluids undergoing capillary break-up in air. This framework is further extended to encompass the effect of outer viscous fluids. As a result, we present a two-dimensional operating map in which the boundaries are set by fluid properties and a single geometrical parameter, related to the experimental configuration. This approach establishes guidelines to correctly interpret experimental data and identify transitions in capillary break-up experiments of liquid bridges surrounded by fluids of different viscosities.
\end{abstract}

DOI: 10.1103/PhysRevFluids.5.051901

Capillary break-up has been extensively employed in the past as a tool to study the thinning behavior of complex fluid bridges and to extract material properties in extensional flows such as the transient (apparent) extensional viscosity, or the longest relaxation time [1,2]. Nonetheless, a successful application of this technique relies on a proper identification of the underlying dynamics, originating either from a balance or dominance of single material properties that causes the minimum liquid bridge radius $R_{\min }$ to exhibit a certain scaling $R_{\min }=H f(t)$, with $f(t)$ some function of time $t$. In most cases, similarity solutions were needed to determine the necessary numerical prefactors $H$ for a quantitative evaluation of a scaling regime [3]. A multitude of dynamical regimes and $f(t)$, such as viscocapillary (V) [4], elastocapillary (EC) [5], inertia capillary (IC) [6,7], and inertia viscous (IV) [8], have been identified in the literature based on the relevant force balance. Additional regimes were highlighted in more complex scenarios, for instance, when the viscosity of the surrounding fluid cannot be neglected [two-fluid viscous (LV) and line-sink flow (LSF) regimes [9,10]], for bubbles undergoing capillary thinning in fluids of various viscosities [11] or for confined flows [12]. A comprehensive description can be found in Refs. [1,13,14].

*These authors contributed equally to this work. 
Intensive theoretical studies, usually based on scaling arguments, were carried out to formulate criteria that allow one to identify the presence of these regimes and their transitions $[13,15]$. This is in particular necessary for regimes that exhibit the same $f(t)$ [as the linear V, IV, and LV (LSF) regimes], so that an experimentally observed scaling is not sufficient for their identification. However, Eggers and Villermaux [13] address that discrepancies exist between proposed criteria for $\mathrm{V}$ to IV transitions and experimental observations $[16,17]$, possibly depending on the overall geometry, which can also be seen in Ref. [18]. One cause lies in limitations to access criteria that focus on the thinning stages close to break-up, hence close to or below (optical) resolution limits. Second, effective criteria based on local velocities, local gradients, or Reynolds numbers [19] require information that is hardly experimentally available and rely on numerical calculations. Third, and most important, the experimental axial length scales of the liquid bridge have so far not been taken into account. Although case-specific geometrical parameters were employed to determine global behavior and regime transitions, they are usually buried in prefactors and arbitrary constants.

The purpose of this Rapid Communication is therefore to establish a systematic framework to identify transition criteria between linear thinning regimes in liquid bridges, solely based on material properties and characteristic dimensions of different experimental setups. To this end, we introduce in the following a set of nondimensional groups based on the experimentally relevant axial length scale of the liquid bridge. The concept of an axial Ohnesorge number $\mathrm{Oh}_{\mathrm{L}}$, along with the nondimensional radius $a$, is initially introduced for Newtonian, viscous-dominated fluids undergoing capillary break-up in air. Subsequently, the analysis is extended to encompass the effect of outer fluids of significant viscosity. Experimental limits, which depend on the setup configuration, are employed to identify the observable regimes and their transitions. As a result, a two-dimensional (2D) operating map is introduced, in which the boundaries are set by our dimensionless groups and the dependent scaling relations. This map is an alternative guideline to identify regimes and interpret experimental observations for viscous liquids surrounded by air and fluids of different viscosities.

For Newtonian fluids, the balance controlling the radial thinning behavior has so far been given in terms of the global Ohnesorge number $\mathrm{Oh}=\eta_{\text {in }} / \sqrt{\gamma \rho R}$ that compares radial viscous and inertial contributions (where $\eta_{\text {in }}$ is the shear viscosity, $\gamma$ the surface tension, $\rho$ the density, and $R$ the characteristic radial dimension of the liquid bridge). For a viscous-dominated thinning (V regime), Papageorgiou [4] exploited a similarity solution to show that the prefactor in the linear decay of the minimum radius of a slender liquid bridge $R_{\min } \sim-H \gamma t / \eta_{\text {in }}$ takes on the value $H=0.0709$ [20]. As the liquid bridge thins, inertia becomes progressively more significant [21], leading eventually to a transition to the also linear inertia-viscous regime (IV), for which $H=0.0304$ [8]. However, as indicated above, a transition criterion between the V and IV regime that scales with Oh [13] deviates up to orders of magnitude for different experimental setups. We propose that the underlying difference between the experiments is the length $L$ of the liquid bridge, which is so far missing in the dimensional analysis. Including an axial length scale $L$, it is straightforward to show that the Buckingham $\pi$ theorem yields as one possible solution for the now two alternative groups

$$
\mathrm{Oh}_{\mathrm{L}}=\frac{\eta_{\text {in }}}{\sqrt{\gamma \rho L}}, \quad a=\frac{R}{L},
$$

and thus as a transition criterion $\mathrm{Oh}_{\mathrm{L}}^{2} a$ that can also be derived balancing axial inertial and viscous stresses. Both groups now incorporate with the axial length scale $L$ an unconventional nondimensionalization of the fluid properties that captures, however, the essential geometrical differences between experimental setups, which was generally not possible with the traditional nondimensionalization using an often arbitrary initial radial dimension $R$. In the most commonly used capillary break-up techniques, the liquid bridge is held between two fixed boundaries: in capillary break-up extensional rheometry (CaBER) experiments [20] between circular plates with adjustable distances of $\mathrm{O}(1 \mathrm{~cm})$, in a Rayleigh-Ohnesorge jetting extensional rheometer (ROJER) [22] between equidistant $[\mathrm{O}(100 \mu \mathrm{m})]$ droplets on a flying jet, while dripping-onto-substrate 
extensional rheometry (DoS) [23] probes a liquid bridge of $\mathrm{O}(1 \mathrm{~mm})$ confined between a nozzle and a substrate. Contrary to unconfined liquid filaments, extensively studied by Schulkes, Basaran, and Hutchings [24-27], the liquid bridge length (and thus $L$ ) is fixed for a capillary break-up experiment [13], but differs up to two orders of magnitude between the different configurations.

The significance of the axial length scale is supported by a transition criterion between the $\mathrm{V}$ and IV regime recently introduced by $\mathrm{Li}$ and Sprittles [28], which they suggested to take place when the local Reynolds number $\operatorname{Re}=\rho u L / \eta_{\text {in }}$ (where $u$ is the local axial velocity at an axial distance $L$ away from the point of minimal axial velocity) reaches a critical value of $\operatorname{Re}=0.85$ at the point of maximum axial velocity $u_{\max }$ (at $L_{\max }$ ). One can decouple Re (and conveniently avoid the experimentally difficult to access local velocity) into $\mathrm{Oh}_{\mathrm{L}}$ and $a$ by approximating the slender liquid bridge with a cylindrical geometry. Replacing in Re the axial velocity via the strain rate $\dot{\varepsilon}=u / L=-2 \dot{R} / R$, using the linear radius decay of Papageorgiou for viscous fluids [4], yields $\operatorname{Re}=\left(2 H \rho \gamma L^{2}\right) /\left(\eta_{\text {in }}^{2} R\right)=0.1418 /\left(\mathrm{Oh}_{\mathrm{L}}^{2} a\right)$.

$V$ to $I V$. We can now determine the critical transition criteria for the dimensionless radius $a$ exclusively based on fluid properties using the axial $\mathrm{Oh}_{\mathrm{L}}$ of Eq. (1), once the critical liquid bridge length scale $L$ is introduced. Without using numerical methods, a critical axial length scale $L$, defined as the axial distance $L_{\max }$ between the point of maximum and zero axial velocity (analogously to Ref. [28]), can be accessed from the velocity profiles derived from the experimentally obtained liquid bridge shapes. In the $\mathrm{V}$ regime, this $L_{\max }$ is predicted to scale with time only as $\sim\left(t_{b}-t\right)^{0.175}[3,4]$, and this near time independence has been numerically [28] and experimentally (see the Appendix) confirmed. Using the numerically determined transition criterion of $\operatorname{Re}=0.85$ [28] would give $\left(\mathrm{Oh}_{\mathrm{L}}^{2} a\right)_{\text {crit }}=0.17$. However, taking into account the experimentally observed deviations from the ideal cylindrical shape via an experimentally observed correction factor of 1.5 (see the Appendix) gives for the transition from $\mathrm{V}$ to IV

$$
a_{\text {crit }}=\frac{R_{\text {crit }}}{L}=\frac{0.11}{\mathrm{Oh}_{\mathrm{L}}^{2}} .
$$

$V$ to $L V$. Next, we apply this alternative nondimensionalization via $L$ to identify the transitional criteria also in the presence of an outer immiscible fluid of significant viscosity, assuming the inner fluid to be incompressible and the density mismatch between the two fluids $\Delta \rho$ insignificant $[29,30]$. Lister and Stone [9] have shown that, even for low viscous outer fluids, the drag exerted by the outer liquid can no longer be neglected when break-up is approached. Introducing the nondimensional viscosity ratio $p=\eta_{\text {in }} / \eta_{\text {out }}$ of inner to outer fluid, for $p \gg 1$ the flow inside the liquid bridge is predominantly a uniaxial extension and causes a radially decaying shear drag on the outer fluid [see Fig. 1(a)]. They approximated this drag as the shear stress induced by a cylinder sliding axially through the outer fluid, yielding the momentum balance [in terms of $a$ of Eq. (1)]

$$
\frac{\gamma}{R}=3 p \eta_{\text {out }} \dot{\varepsilon}-\frac{B \eta_{\text {out }}}{2 a^{2}} \dot{\varepsilon}
$$

where $B=2 /|\ln a|$ is a dimensionless coefficient [9]. The dimensionless group that arises when balancing inner and outer fluid stresses on the right-hand side of Eq. (3) is $p a^{2}|\ln a|$, which describes the transition from the $\mathrm{V}$ regime, where viscous stresses generated by the outer fluid are negligible, to one in which they control the thinning behavior (LV). Again, we can describe the transition in terms of a critical radius,

$$
a_{\text {crit }}^{2}\left|\ln a_{\text {crit }}\right|=\frac{1}{3 p}
$$

$L S F$ to $L V$. As pointed out by Sierou and Lister [10], a drastically different physical picture is seen for small viscosity ratios $(p \ll 1)$. In the outer fluid, the flow is a nearly pure radial extension, resembling a line distribution of sinks, while a parabolic shear (Poiseuille) flow occurs in the inner 

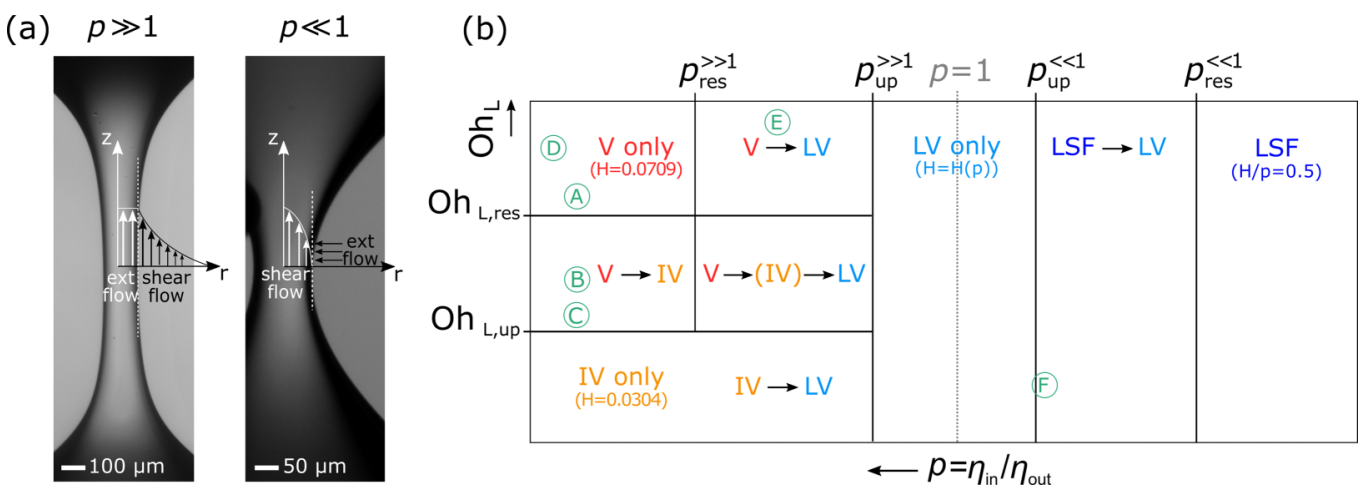

FIG. 1. (a) High-resolution images from CaBER experiments performed with surrounding immiscible outer liquids. The schematics highlights the differences in the expected inner and outer flow profiles for $p=$ $18.7 \gg 1$ and $p=0.002 \ll 1$, respectively. (b) $2 \mathrm{D}$ operating map as a function of the dimensionless groups $\mathrm{Oh}_{\mathrm{L}}$ and $p$. The derived criteria are used to delimit the areas within which specific regimes and transitions can be experimentally detected. Circled green letters correspond to the capillary break-up experiments for various $L$, and inner and outer fluid viscosities of Fig. 2.

liquid, as illustrated in Fig. 1(a). The momentum balance takes on the form

$$
\frac{\gamma}{R}=\frac{4 u p \eta_{\text {out }} L}{R^{2}}+\eta_{\text {out }} \frac{u}{L}
$$

in which $u$ is the radially averaged velocity in the axial direction in the inner fluid. Balancing the two terms on the right-hand side in Eq. (5) establishes the transition from a thinning regime dominated by the radial drag of the outer fluid [line-sink flow (LSF)] to the point where viscous friction of the inner fluid can no longer be neglected (LV), and determines a dimensionless group $p / a^{2}$. The critical transition criterion can then be rewritten as

$$
a_{\text {crit }}=\sqrt{4 p} \text {. }
$$

Observation limits for transitions. The transitional criteria for $a$ introduced above are defined solely based on material properties and one geometrical parameter via the dimensionless groups $\mathrm{Oh}_{\mathrm{L}}$ and $p$. It is crucial to note that an $a_{\text {crit }}$ can vary significantly for the same fluid due to the intrinsically different axial dimensions and length scales $L$ employed in the various capillary break-up techniques CaBER [20], DoS [23], and ROJER [22]. This also entails that the same regimes and transitions might not necessarily be observable in capillary break-up experiments performed on the same fluid, but with different techniques (and thus different $L$ ). However, since $L$ is observed to not change significantly in time in the $\mathrm{V}$ regime, this geometry-dependent length scale can be used to assess the observation range for different setups. The derived transitional criteria can be reworked as

$$
\mathrm{Oh}_{\mathrm{L}}=\sqrt{\frac{0.11}{a_{\text {crit }}}}, \quad p^{\gg 1}=\frac{\left|\ln \left(a_{\text {crit }}\right)\right|^{-1}}{3 a_{\text {crit }}^{2}}, \quad p^{\ll 1}=\frac{a_{\text {crit }}^{2}}{4} .
$$

Setting $a_{\text {crit }}$ equal to the upper and lower observation limits of the radius for a given $L$ of a setup allows us to determine a priori for which $\mathrm{Oh}_{\mathrm{L}}, p^{\gg 1}$, or $p^{\ll 1}$ an $a_{\text {crit }}$ can be experimentally observed. Since most regimes are based on a slenderness assumption (i.e., $a=R / L \ll 1$ ), a first limit is set by an upper radius $R_{\text {up }}$, so that $a_{\text {crit }} \leqslant a_{\text {up }}=R_{\text {up }} / L$ in order for a transition to be observable. Using Eq. (7), $a_{\text {up }}$ identifies then the critical global $\mathrm{Oh}_{\mathrm{L} \text {,up }}$ below which no transition but only the IV regime is observed. Similarly, the upper slenderness limit $a_{\text {up }}$ can also be used to determine the viscosity ratio $p_{\text {up }}^{\gg 1}$ below which only the LV regime is visible for a set $L$. An upper criterion for $p \ll 1$ can, however, not be derived based on the same argument in a straightforward fashion. Due to the quadratic nature of the liquid bridge profile in the LSF regime [32], $L$ is much smaller compared to 
the other cases [see, for example, the image for $p \ll 1$ in Fig. 1(a)] and the bridge only becomes sufficiently slender when the transition to LV itself takes place. Nonetheless, a slenderness-based limit $p_{\text {up }}^{\ll 1}$ can still be used to determine the critical viscosity ratio below which the LSF regime can be detected right before transitioning to the slender LV regime.

The lower critical boundary is set by the radial resolution limit $R_{\text {res }}$, which varies significantly with the experimental detection technique [11] and is usually disregarded in numerical studies. As for the upper limit, from $a_{\text {crit }} \geqslant a_{\text {res }}=R_{\text {res }} / L$ the lower limits $\mathrm{Oh}_{\mathrm{L}, \text { res }}, p_{\text {res }}^{\gg 1}$, and $p_{\text {res }}^{\ll 1}$ are obtained via Eq. (7).

All the aforementioned limits are illustrated in Fig. 1(b) where a 2D map describes the observable regimes and transitions in terms of $\mathrm{Oh}_{\mathrm{L}}$ and $p$ and thus the respective radii (once the fluid properties and in particular the axial length $L$ of the setup are known).

Experimental verification. To verify our introduced criteria and limits, we use the map in Fig. 1(b) to predict the behavior and transitions for a number of experiments encompassing the 2D parameter space. The capillary break-up experiments are performed using a CaBER rheometer (Thermo Haake, high-speed video-imaging re-equipped). $a_{\text {up }}$ is calculated assuming a slenderness limit $L / R=10$ [28], and $a_{\text {res }}$ is calculated based on the limit of the optical setup, which can accurately resolve down to a radius of $5 \mu \mathrm{m}$. The geometrical parameter $L$ is determined following the Appendix, and is experimentally found to be directly related to the final plate separation distance. Water and glycerol-water mixtures are used as inner fluids, and air and silicon oils of different viscosities as outer fluids.

To show the importance of the geometry to determine the onset of the $\mathrm{V}$ to IV transition, in a first series of experiments [A, B, and C in Fig. 1(b)] we move through the map down the vertical axis for a high, fixed value of $p$. The experiments are performed with the same inner fluid $\left(\eta_{\text {in }}=0.365 \mathrm{Pas}, \gamma=65 \mathrm{mNm}\right)$ surrounded by air $(p=24333)$, while the geometrical factor $L$ is changed by varying the final plate distance. The temporal evolution of the minimum radius is shown in Fig. 2(a) for the three cases, together with the thinning predictions for the $\mathrm{V}$ regime $(H=0.0709)$ and the IV regime $(H=0.0304)$. Figure $2(a)$ clearly illustrates how the window between $R_{\text {up }}$ and $R_{\text {crit }}$ for the observation of a transition drastically increases by increasing $L$. Moreover, $R_{\text {crit }}$ markedly corresponds to the radius at which experimental data start deviating from the theoretical $\mathrm{V}$ thinning, thus accurately predicting the onset of a transition to IV. A second series of experiments [D and E in Fig. 1(b)] shows the effect of increasing viscosity of the outer fluid while keeping $L$ and inner fluid properties constant $\left(\eta_{\text {in }}=0.518 \mathrm{Pas}\right.$ ). With air as the outer fluid, case D $(p=34435)$ is comparable to case A, where the transition to IV is below the resolution limit, as shown by the minimum radius evolution in Fig. 2(b). By adding an outer liquid of sufficiently high viscosity ( $p=96$ ), $R_{\text {crit }}$ calculated with Eq. (4) lies now within the experimentally detectable range and nicely corresponds to the experimentally observed onset of the LV regime in Fig. 2(ii). Moving to the other side of the map towards low viscosity ratios, case $\mathrm{F}$ in Fig. 1(ii) $\left(\eta_{\text {in }}=10^{-3} \mathrm{Pas}\right.$, $p=0.002)$ represents an area of the map at the limits of experimental accessibility. Nonetheless, the $R_{\min }$ evolution in Fig. 2(c) shows that Eq. (6) precisely predicts the transition from LSF to LV.

To conclude, in this Rapid Communication, we introduce a theoretical framework for capillary break-up experiments to determine the critical transition radii between dynamical regimes when viscous stresses are governing the inner or outer fluid flows (or both). Our criteria depend solely on fluid properties via an alternative set of nondimensional groups incorporating the axial length scale $L$ of the liquid bridge, which is for slender filaments in the $\mathrm{V}$ regime directly related to the axial dimension of the experimental setup. The criteria describe a $2 \mathrm{D}$ map to be used as a guideline to correctly pinpoint the observability of transitions between linear thinning regimes in experiments, as proven by examples covering a wide parameter range. Still, further experiments are needed to refine the inner part of the parameter space. In particular, the IV to LV transition is indicated to take place at $a \sim p^{-1 / 2}$ [9], but the correlation of the length scale $L$ to the geometrical parameters of the bridge in the IV regime is unexplored. A full comprehension of predominantly viscous fluids will allow us to expand the map to more difficult scenarios, including the presence of 

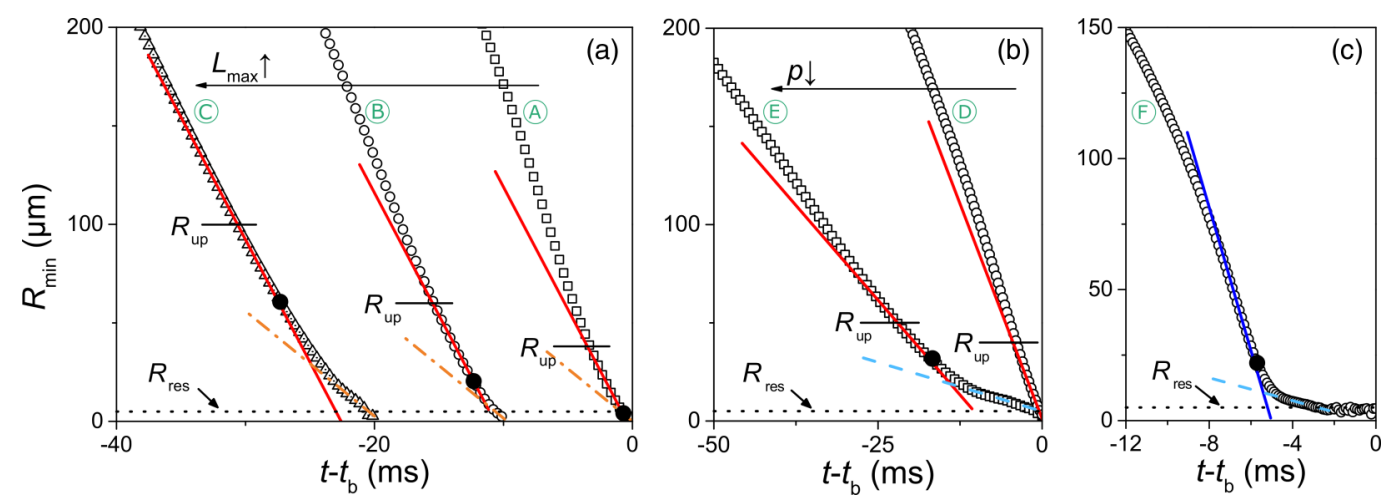

FIG. 2. Minimum radius evolution $\left(R_{\min }\right)$ in time, with $t_{\mathrm{b}}$ as the break-up time, for several fluids and geometrical conditions in CaBER experiments performed in air [(a), (b) (open circles)] and with an outer liquid [(b) (open squares), (c)]. Solid dots represent calculated $R_{\text {crit }}$. Thinning curves in A are shifted $10 \mathrm{~ms}$ apart for clarity. (a) Experimentally detectable regimes are determined by the set geometrical conditions. For the same fluid ( $\eta_{\text {in }}=0.365 \mathrm{Pas}, \gamma=65 \mathrm{mNm}$ ), $R_{\text {crit }}$ via Eq. (2) shifts to larger radii and the experimental window $\left(R_{\text {up }}-R_{\text {res }}\right)$ expands as the geometrical parameter $L$ increases (right to left: 480,600 , and $1000 \mu \mathrm{m}$ ). Consistently, the thinning dynamics appear to be solely $\mathrm{V}$ dominated for the lowest $L$ (open squares), whereas a region following the IV scaling is clearly distinguishable for the highest $L$ (open triangles). $R_{\text {crit }}$ predicted with Eq. (2) matches the deviation of the data from the predicted V scaling $(H=0.0709$, red solid line) towards the IV scaling $\left(H=0.0304\right.$, orange dashed-dotted line). (b) Thinning curves of the same inner fluid $\left(\eta_{\text {in }}=0.518\right.$ Pas, $\gamma=63.7 \mathrm{mN} \mathrm{m}$ and $30.0 \mathrm{mN} \mathrm{m}$ for $\mathrm{D}$ and $\mathrm{E}$, respectively) at comparable geometrical conditions show different behaviours depending on the outer medium viscosity (open circles $p=34435$, open squares $p=96$ ). For $p \gg 1$, the transition from $\mathrm{V}(H=0.0709$, red solid line) to LV regime $[H(p)=0.02$ [31], blue dashed line] is detectable only if $\eta_{\text {out }}$ is sufficiently high to fulfill the condition $p_{\text {res }} \ll p \ll p_{\text {up }}$. (c) For $p=0.002$, $R_{\text {crit }}$ according to Eq. (4) correspond to the deviation from $\operatorname{LSF}(H=0.5$, blue solid line) to the LV regime $[H(\mathrm{p})=0.04[10]$, blue dashed line $]$.

viscoelasticity, for which the operating space could be expanded to 3D by introducing the Deborah (De) or Weissenberg (Wi) numbers.

Acknowledgments. The authors would like to acknowledge financial support from the Fonds Wetenschappelijk Onderzoek - Vlaanderen (FWO, Grant No. G077916N), as well as from the Bijzonder Onderzoeksfonds KU Leuven (GOA 15/007).

\section{APPENDIX}

To decouple $\mathrm{Re}$ into $\mathrm{Oh}_{\mathrm{L}}$ and $a$, the liquid bridge was approximated as a perfect cylinder for which the axial velocity $u=\dot{L}$ increases linearly along the bridge axis. Nonetheless, the self-similar profiles of actual fluid bridges exhibit an axial curvature [Fig. 3(a)], which causes a significant deviation of $u(z)$ from linearity. Here, we introduce an experimental method to determine the radially averaged axial velocity $u_{\mathrm{a}}(z, t)$, a numerical correction factor $C$ accounting for this deviation for the determination of $a_{\text {crit }}$ [Eq. (2)], and the length $L_{\max }$ at $u_{\mathrm{a}, \max }$.

$u_{\mathrm{a}}(z, t)$ [Fig. 3(b)] can directly be obtained from the experimentally observed evolution of the bridge shape, following the approach of Rothert et al. [16]. For this the cumulative volume $V_{\text {cumul }}\left(z=z_{0}+i \Delta z, t\right)=\sum_{z_{0}}^{z_{0}+i \Delta z} \Delta z \pi R^{2}\left(z_{0}+i \Delta z, t\right)$ is determined from the radius evolution [with $z_{0}(t)$ the position of the minimum radius, and $\Delta z$ the pixel size]. Assuming zero axial velocity at $z_{0}(t)$, the axial velocity at position $z$ is

$$
u_{\mathrm{a}}(z, t)=\frac{1}{\pi R^{2}(z, t)} \frac{d V_{\text {cumul }}(z, t)}{d t} .
$$



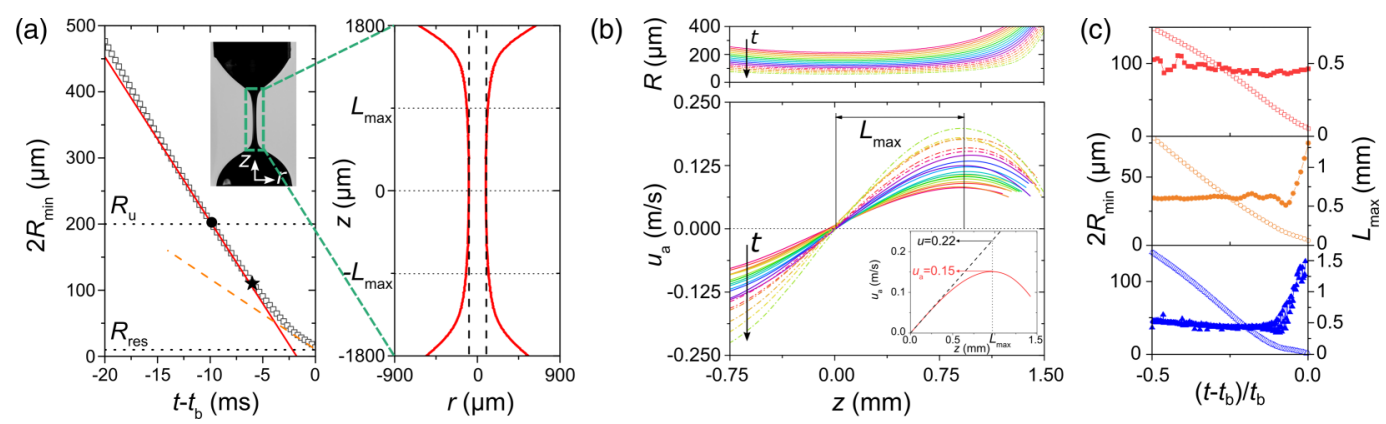

FIG. 3. (a) Capillary thinning evolution of a Newtonian fluid with $\eta_{\text {in }}=0.365 \mathrm{mPa}$ s in air and with $L_{\max }=900 \mu \mathrm{m}$. $R_{\text {crit }}$ calculated from the linear evolution of $u$ (black solid dot, high-resolution image in the inset) overestimates the V to IV transition, which is instead correctly captured by $R_{\text {crit }}$ calculated incorporating the corrective factor $C$ in Eq. (2) (black solid star). The enlargement of the inset shows the visible difference between the ideal cylinder geometry (black dashed lines) and the actual profile (red solid lines). (b) Temporal evolution of $R(\mathrm{z})$ and $u_{\mathrm{a}}(\mathrm{z})$ in a capillary break-up experiment of a glycerol-water mixture in air ( $\eta_{\text {in }}=0.365 \mathrm{mPas}$ ) with $L_{\max }=900 \mu \mathrm{m}\left(-18.33 \mathrm{~ms}<t-t_{\mathrm{b}}<-6.33 \mathrm{~ms}\right.$ at $\left.\Delta t=0.66 \mathrm{~ms}\right)$. Inset: The deviation of the actual profile from the ideal cylinder geometry implicates a large difference at $L_{\max }$ between $u_{\mathrm{a}}=0.15 \mathrm{~m} / \mathrm{s}$ (red solid line) and $u=0.22 \mathrm{~m} / \mathrm{s}$ (black dashed line) calculated assuming a linear evolution of the axial velocity. (c) Direct comparison between the temporal evolution of $R_{\min }$ (open symbols) and $L_{\max }$ (solid symbols), determined from the experimental $u_{\mathrm{a}}$ profiles. The characteristic length remains approximately constant throughout the complete bridge evolution for case A of Fig. 2 (top, red squares). Also for cases $\mathrm{B}$ (middle, orange circles) and $\mathrm{E}$ (bottom, blue triangles) $L_{\max }$ remains constant in the $\mathrm{V}$ regime, up to the transitions to the asymmetrical thinning regimes (IV or LV).

Due to axial curvature at the bridge end bulges, the actual average axial velocity profile exhibits a sinusoidal shape [Fig. 3(b)], allowing us to determine a $u_{\max }$ at $L_{\max }$. Plotting $L_{\max }(t)$ in Fig. 3(c) shows that, within the $\mathrm{V}$ regimes, $L_{\max }$ remains approximately constant for different experimental conditions of Fig. 2. The inset in Fig. 3(b) shows the overestimation of the velocity at $z=L_{\max }$ when assuming a linear increase of $u$ with $z$. Evidently, also the calculated $R_{\text {crit }}$ in Fig. 3 (solid dot, calculated from $a_{\text {crit }}=R_{\text {crit }} / L_{\text {max }}=0.17 \mathrm{Oh}_{\mathrm{L}}^{2}$ ) lies then above the actual transition radius compared to experimental data, while the theoretical V and IV scalings (red solid and orange dashed lines, respectively) suggest a much later transition. To correct for this overestimation, $C=u_{\text {act }} / u$ at $L_{\max }$ has been calculated for several CaBER experiments performed on V-dominated fluids at different $L$. As expected for self-similar thinning, $C$ assumes a constant value of 1.5 , and is used to quantitatively correct the criterion derived for $a_{\text {crit }}$ [Eq. (6)]. The corrected $R_{\text {crit }}$ [Fig. 3(a), black solid star] corresponds then to the deviation from the V scaling, indicating the transition to the IV regime.

[1] G. H. McKinley, Visco-elasto-capillary thinning and break-up of complex fluids, Rheol. Rev. 3, 1 (2005).

[2] C. Clasen, P. M. Phillips, L. Palangetic, and J. Vermant, Dispensing of rheologically complex fluids: The map of misery, AIChE J. 58, 3242 (2012).

[3] J. Eggers, Nonlinear dynamics and breakup of free-surface flows, Rev. Mod. Phys. 69, 865 (1997).

[4] D. T. Papageorgiou, On the breakup of viscous liquid threads, Phys. Fluids 7, 1529 (1995).

[5] V. Entov and E. Hinch, Effect of a spectrum of relaxation times on the capillary thinning of a filament of elastic liquid, J. Non-Newtonian Fluid Mech. 72, 31 (1997).

[6] Y. Chen and P. Steen, Dynamics of inviscid capillary breakup: Collapse and pinchoff of a film fridge, J. Fluid Mech. 341, 245 (1997).

[7] R. F. Day, E. J. Hinch, and J. R. Lister, Self-Similar Capillary Pinchoff of an Inviscid Fluid, Phys. Rev. Lett. 80, 704 (1998). 
[8] J. Eggers, Universal Pinching of SD Axisymmetric Free-Surface Flow, Phys. Rev. Lett. 71, 3458 (1993).

[9] J. R. Lister and H. A. Stone, Capillary breakup of a viscous thread surrounded by another viscous fluid, Phys. Fluids 10, 2758 (1998).

[10] A. Sierou and J. R. Lister, Self-similar solutions for viscous capillary pinch-off, J. Fluid Mech. 497, 381 (2003).

[11] J. C. Burton, J. E. Rutledge, and P. Taborek, Fluid Pinch-Off Dynamics at Nanometer Length Scales, Phys. Rev. Lett. 92, 244505 (2004).

[12] A. A. Pahlavan, H. A. Stone, G. H. McKinley, and R. Juanes, Restoring universality to the pinch-off of a bubble, Proc. Natl. Acad. Sci. USA 116, 13780 (2019).

[13] J. Eggers and E. Villermaux, Physics of liquid jets, Rep. Prog. Phys. 71, 1 (2008).

[14] S. L. Anna, Droplets and bubbles in microfluidic devices, Annu. Rev. Fluid Mech. 48, 285 (2015).

[15] O. A. Basaran, Small-scale free surface flows with breakup: Drop formation and emerging applications, AIChE J. 48, 1842 (2002).

[16] A. Rothert, R. Richter, and I. Rehberg, Formation of a drop: Viscosity dependence of three flow regimes, New J. Phys. 5, 59 (2003).

[17] A. Rothert, R. Richter, and I. Rehberg, Transition from Symmetric to Asymmetric Scaling Function before Drop Pinch-Off, Phys. Rev. Lett. 87, 084501 (2001).

[18] W. Mathues, C. McIlroy, O. G. Harlen, and C. Clasen, Capillary breakup of suspensions near pinch-off, Phys. Fluids 27, 093301 (2015).

[19] J. R. Castrejón-Pita, A. A. Castrejón-Pita, S. S. Thete, K. Sambath, I. M. Hutchings, J. Hinch, J. R. Lister, and O. A. Basaran, Plethora of transitions during breakup of liquid filaments, Proc. Natl. Acad. Sci. USA 112, 4582 (2015).

[20] G. H. McKinley and A. Tripathi, How to extract the Newtonian viscosity from capillary breakup measurements in a filament rheometer, J. Rheol. 44, 653 (2000).

[21] M. P. Brenner, J. R. Lister, and H. A. Stone, Pinching threads, singularities and the number 0.0304, Phys. Fluids 8, 2827 (1996).

[22] V. Sharma, S. J. Haward, J. Serdy, B. Keshavarz, A. Soderlund, P. Threlfall-Holmes, and G. H. McKinley, The rheology of aqueous solutions of ethyl hydroxy-ethyl cellulose (EHEC) and its hydrophobically modified analogue (hmEHEC): Extensional flow response in capillary break-up, jetting (ROJER) and in a cross-slot extensional rheometer, Soft Matter 11, 3251 (2015).

[23] J. Dinic, Y. Zhang, L. N. Jimenez, and V. Sharma, Extensional relaxation times of dilute, aqueous polymer solutions, ACS Macro Lett. 4, 804 (2015).

[24] A. A. Castrejón-Pita, J. R. Castrejón-Pita, and I. M. Hutchings, Breakup of liquid filaments, Phys. Rev. Lett. 108, 074506 (2012).

[25] P. K. Notz and O. A. Basaran, Dynamics and breakup of a contracting liquid filament, J. Fluid Mech. 512, 223 (2004).

[26] R. M. S. M. Schulkes, The contraction of liquid filaments, J. Fluid Mech. 309, 277 (1996).

[27] C. R. Anthony, P. M. Kamat, M. T. Harris, and O. A. Basaran, Dynamics of contracting filaments, Phys. Rev. Fluids 4, 093601 (2019).

[28] Y. Li and J. E. Sprittles, Capillary breakup of a liquid bridge: Identifying regimes and transitions, J. Fluid Mech. 797, 29 (2016).

[29] J. Eggers and T. F. Dupont, Drop formation in a one-dimensional approximation of the Navier-Stokes equation, J. Fluid Mech. 262, 205 (1994).

[30] I. Cohen and S. R. Nagel, Testing for scaling behavior dependence on geometrical and fluid parameters in the two fluid drop snap-off problem, Phys. Fluids 13, 3533 (2001).

[31] I. Cohen, M. P. Brenner, J. Eggers, and S. R. Nagel, Two Fluid Drop Snap-Off Problem: Experiments and Theory, Phys. Rev. Lett. 83, 1147 (1999).

[32] P. Doshi, I. Cohen, W. W. Zhang, M. Siegel, P. Howell, O. A. Basaran, and S. R. Nagel, Persistence of memory in drop breakup: The breakdown of universality, Science 302, 1185 (2003). 